\title{
Enfoque algorítmico para el cálculo del desgaste en pares de fricción
}

\section{de materiales metálicos $(\cdot)$}

\author{
Francisco Martínez Pérez*
}

\begin{abstract}
Resumen Las propiedades tribológicas de los materiales de los pares de fricción constituyen, hoy, parte de los requerimientos tecnológicos exigidos, por tener una influencia decisiva en la vida útil de estos pares. Un parámetro esencial a medir es el desgaste que sufren los materiales en su trabajo. Estas mediciones pueden efectuarse en pruebas de campo o laboratorio, pero también puede calcularse este desgaste y para ello hay que tener en cuenta el mecanismo actuante. En el trabajo se expone el desarrollo de un algoritmo para el cálculo del desgaste, que recoge y sintetiza las recomendaciones principales que aparecen en la diversa literatura especializada.
\end{abstract}

Palabras clave Desgaste. Tribología. Fricción.

\section{Algorithm approach for wear calculation of friction pairs of metallic materials}

\begin{abstract}
Tribological properties of since materials for friction pairs are nowadays part of the tecnological requirements they have a decisive influence on the useful life of these pairs. An essential parameter to be measured is the wear undergone by materials that is caused durin their perfomance. These measures can be taken in both lab or field testing, but they can be also calculated. For this, the wear mechanism must be taken into account. In the present paper, an algorithm for the calculation of wear based on the main disperse recommendations from the specializad literature is given.
\end{abstract}

Keywords Wear. Tribology. Friction.

\section{INTRODUCCIÓN}

La aplicación de la tribología como tecnología que permite influir en la reducción de las pérdidas por fricción y las de desgaste en los pares de fricción, es hoy introducida en países desarrollados como Estados Unidos, Inglaterra, Japón, Francia, Alemania, Rusia y otros. Algunos la introducen como un requerimiento a cumplir por las superficies interactuantes durante su trabajo.

Muchos investigadores de estos países han trabajado en formulaciones que permitan calcular y predecir el desgaste. Estas formulaciones se encuentran dispersas en las publicaciones efectuadas, ya que, dependiendo del mecanismo de desgaste y de la presencia de "terceros cuerpos", habrá una incidencia diferenciada en los principales parámetros a tener en cuenta para el cálculo del desgaste.

Por otra parte, el diseñador o especialista en mantenimiento que pretenda hacer estos cálculos no encontrará un ordenamiento que le simplifique su labor.

En el trabajo, después de tener en cuenta las formulaciones para el cálculo del desgaste en la literatura especializada, se presenta a consideración un algoritmo que pretende simplificar este trabajo para aquellos que laboran en el diseño o mantenimiento o para investigadores que lo requieran.

El algoritmo tiene en cuenta los diferentes mecanismos de desgaste y discrimina entre superficies que deslizan una respecto a otra con fricción seca o lubricada. En cada caso se brindan las principales ecuaciones o procedimientos que pueden ser empleados.

\section{DESARROLLO}

En la figura 1 se muestra un enfoque algorítmico para llevar a cabo cálculos del desgaste que sufren pares de fricción de materiales metálicos, sometidos a diferentes mecanismos de desgaste.

(•) Trabajo recibido el día 12 de mayo de 1999 y aceptado en su forma final el día 6 de octubre de 2000.

$\left(^{*}\right)$ Centro de Investigaciones Metalúrgicas, Ministerio de la Industria Sideromecánica y Electrónica. Avenida 51 \# 23611 entre 236 A y 240 , La Lisa, Ciudad Habana, Cuba.Telefax: (537) 336155.E-mail:cime@colombus.cu 


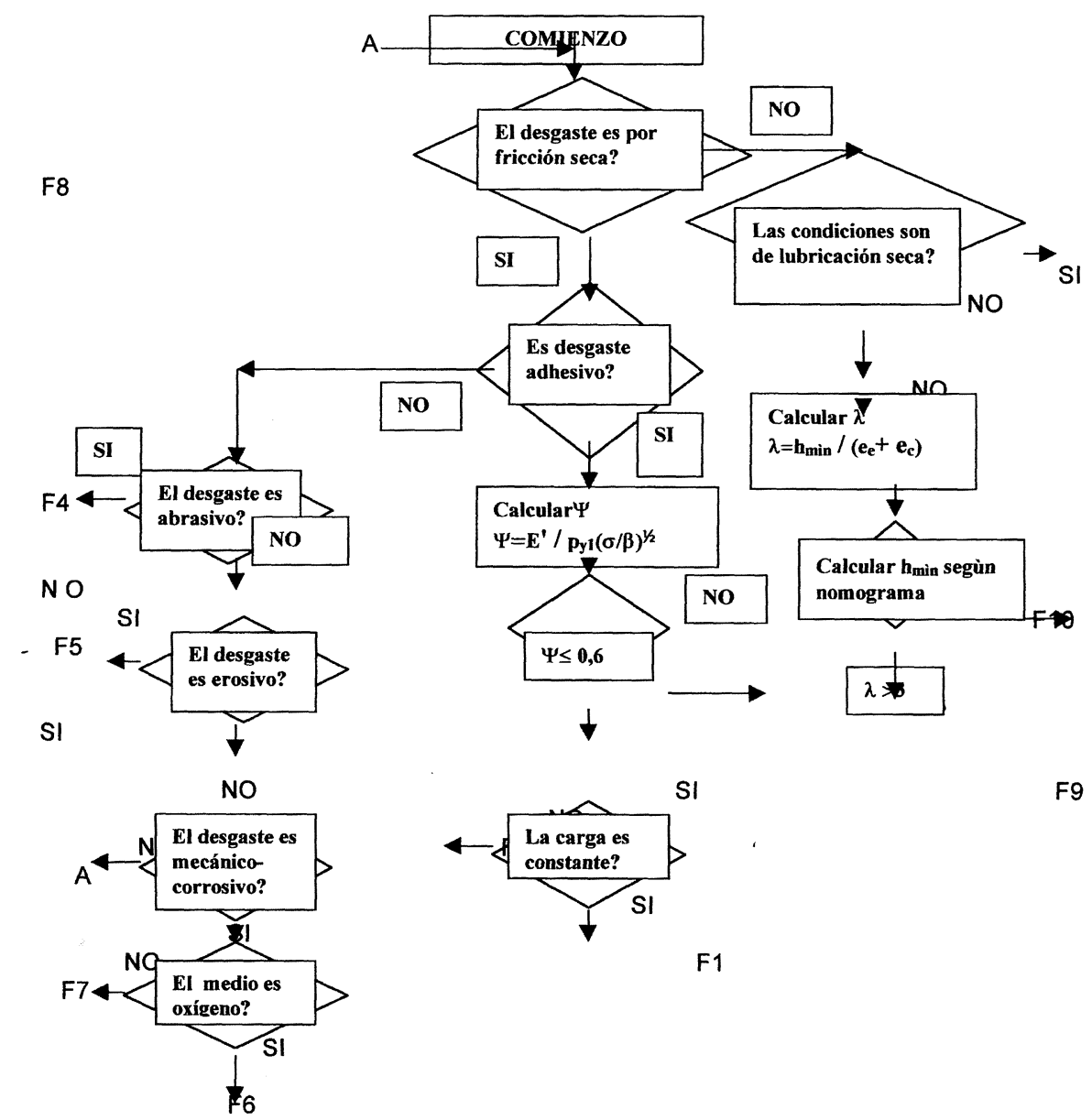

Figura 1. Algoritmo para el cálculo del desgaste en pares de fricción metálicos, en función del tipo de desgaste.

Figure 1. Algorithm for wear calculation on metallic friction joints, depending on the type of wear.

En todo proceso de interacción entre dos superficies siempre habrá que considerar la presencia de un "tercer cuerpo" ya sea ésta casual (medio ambiente) o provocada (capa de lubricante). En el primer caso, y cuando el medio, aunque casual no ejerza condiciones lubricantes (como puede ser la presencia de arcilla en el desgaste abrasivo en suelos) se denominará al proceso de desgaste, de fricción seca. El esquema comienza diferenciando esto. Si la fricción es seca entonces se discrimina el tipo de desgaste, comenzando por el desgaste adhesivo.

\subsection{Desgaste adhesivo}

Si el desgaste es adhesivo, se introduce el concepto de índice de plasticidad, y, que resulta muy conveniente para conocer si el proceso de desgaste ocurre con interacción elástica o plástica entre las asperezas de las superficies ${ }^{[1]}$. El índice y se calcula según la expresión:

$$
\psi=E^{\prime} /\left[p_{\mathrm{y} 1}(\sigma / \beta)^{1 / 2}\right]
$$

donde $E^{\prime}$ es el módulo elástico efectivo, que se obtiene mediante la fórmula:

$E^{\prime}=\left[\left(1-\mu_{1}^{2}\right) / E_{1}+\left(1-\mu_{2}^{2}\right) / E_{2}\right]$, en la que:

$\mu$ es el coeficiente de Poisson del material

E es el módulo elástico del material

$p_{y}$ es la presión que provoca la fluencia en el material y que en ocasiones puede ser sustituida por la dureza $H$

$\sigma$ es la raíz cuadrada de la media de las desviaciones normales de la distribución de la altura de las irregularidades (rms) en las superficies de los dos cuerpos, es decir,

$$
\sigma=\sqrt{\sigma_{1}^{2}+\sigma_{2}^{2}}
$$


$\beta$ es la media del radio de las asperezas

y los subíndices 1 y 2 corresponden al material más blando y más duro, respectivamente, del par de fricción.

Si el cálculo obtiene un valor de $\psi \leq 0,6$, el contacto es de tipo elástico. En este caso habrá que diferenciar, para efectuar los cálculos de desgaste, la forma de accionar de la carga y si ésta es o no constante, dando lugar en el primer caso a la salida F1 y en el segundo, a la salida F2.

\subsubsection{F1: Desgaste adhesivo con interaccion elastica y carga constante}

La intensidad específica de desgaste, $i_{\mathrm{h}}$, viene dada por la expresión:

$$
i_{\mathrm{h}}=x /\left(n_{\mathrm{c}} \sqrt{h / r}\right)
$$

donde:

$x$, factor de intersección entre dos superficies, puede calcularse mediante la fórmula:

$x=[1 / 2(v+1)] \sqrt{v / 2 \gamma}$

en la que

$v$ puede asumirse para el contacto elástico entre $1 \mathrm{y}$ 3 o calcularse, preferiblemente, según la fórmula:

$\nu=1,1\left(R_{\mathrm{p}} / R_{\mathrm{a}}\right)-1$

donde

$R_{\mathrm{p}}$ es la altura media de las rugosidades mayores, es decir,

$R_{\mathrm{p}}=\frac{\sum_{\mathrm{i}=1}^{\mathrm{i}=\mathrm{n}} R \mathrm{p}_{\mathrm{i}}}{\mathrm{n}}$

$R_{\mathrm{a}}$ es la media aritmética de la desviación del perfil, esto es,

$R_{\mathrm{a}}=1 / \mathrm{L} \int_{0}^{\mathrm{L}}[\mathrm{y}(x)] d x$

$\gamma$ es el ángulo de interacción y penetración de la superficie dura en la más blanda y en radianes y se calcula así:

$\gamma=k \frac{N \cdot V}{H_{1}}$ siendo:

$N$ la carga actuante

$V$ la velocidad de deslizamiento

$\mathrm{H}_{1}$ la dureza del material más blando y

$k=1,5 \sqrt{4\left[1-\mu_{1}-\left(\mu_{1}\right)^{2}+\left[\left(1-2 \mu_{1}\right)^{2} / f^{2}\right]\right.}$

fórmula en la que

$\mu_{1}$ es el coeficiente de Poisson del material más blando y

$f$ es el coeficiente de fricción

$n_{c}$, número de ciclos hasta el daño, en la expresión

(2) se calcula según la fórmula:

$n_{\mathrm{c}}=\left[\left(3 \pi \sigma_{\mathrm{el} 1} \theta / 4 k f\right) \sqrt{r / h}\right]^{\mathrm{t}}$

en la que

$\sigma_{\mathrm{el} 1}$ es el límite elástico del material más blando

$\theta=\left(1-\mu_{1}\right)^{2} / E_{1}$ y es la constante elástica del material más blando.

siendo

t una constante que varía

para aceros, de 6 a 9

para fundiciones, de 4 a 5

para bronces, de 4 a 7

$r$ el radio medio de las asperezas del material $h$ la altura media de las cinco asperezas mayores del material más duro.

- El área real de contacto Ar puede ser calculada según Tabor ${ }^{[3]}$ como

$$
A_{r}=\left(F_{n} / E^{\prime}\right)^{n}
$$

siendo:

$F_{n}$ la fuerza normal aplicada

$\mathrm{n}$ un parámetro adimensional donde $2 / 3<n<1$

- La intensidad de desgaste, Ih, puede ser calculada según Kragelski y Kombalov ${ }^{[2]}$ por medio de la fórmula 
$I_{\mathrm{h}}=\frac{\alpha b \varepsilon^{\mathrm{n}+1} R_{\text {máx }}}{(v+1) n d} \eta_{\mathrm{c}, \mathrm{a}}$

donde:

$\alpha$ es un parámetro que en el contacto elástico es 0,5 $b$ es un parámetro que caracteriza la superficie de apoyo y se calcula según la fórmula

$b=0,55\left(R_{\text {máx }} / R_{\mathrm{p}}\right)^{\mathrm{v}}$

$\mathrm{R}_{\text {máx }}$ la altura máxima de las rugosidades

$d=\left(\frac{2 \alpha r R_{\text {máx }} b}{v}\right)^{1 / 2}$

$\eta_{\mathrm{c}, \mathrm{a}}=\operatorname{Ar} / \mathrm{Aa}$

$\varepsilon$ es la aproximación relativa de los cuerpos expresada como la distancia entre $R z_{1}$ y $R z_{2}$, suponiendo el apoyo de los cuerpos en $\mathrm{R}_{\text {máx1 }}$ y $\mathrm{R}_{\text {máx2 } 2}$.

\subsubsection{F2: desgaste adhesivo con interacción elástica y carga variable}

- La intensidad del desgaste Ih se calcula según Kragelski y Kombalov ${ }^{[2]}$ mediante la expresión

$I_{h}=C\left(p_{a}^{\theta} / k_{v}^{\prime}\right)^{t+\beta t} \Delta^{t(1-\beta) / 2}\left(K_{f} / \sigma_{e l}\right)^{t} \eta_{c, a}^{-\beta t} \quad y$

donde

$k_{\mathrm{v}}^{\prime}=k_{\mathrm{v}} / 2 \sqrt{\pi}$

siendo

$k_{v}=\Gamma(v+1) / \Gamma(v+3 / 2)$

$p_{a}$ es la presión nominal de contacto.

C se calcula mediante la expresión

$$
C=\frac{v^{1 / 2} \Gamma(o) \Gamma(1+t / 2)}{4(v+1) \Gamma(v+t / 2)}
$$

$$
\begin{aligned}
& \beta=\frac{1}{2 v+1} \\
& \Delta=\frac{R_{\text {máx }}}{r b^{1 / v}} \\
& K_{f}=\sqrt{\left.\sigma_{\mathrm{el}}-2 f \cdot H B\right) /\left(\sigma_{\mathrm{el}}+2 f \cdot H B\right) v}
\end{aligned}
$$

y

$v=\frac{R_{\mathrm{p}}}{R_{\mathrm{a}}}-1$

$n_{c}$, número de ciclos hasta el daño ${ }^{[2]}$, se calcula según la fórmula:

$n_{c}=\left(\frac{3 \pi \theta \sigma_{\mathrm{el}}}{4 \cdot \mathrm{k} \cdot \mathrm{f}} \sqrt{r / R_{\text {máx }} \varepsilon}\right)^{\mathrm{t}}$

- El área real de contacto puede ser calculada según lo indicado anteriormente en la expresión

Si $\psi>0,6$ el contacto es de tipo plástico

\subsubsection{F3: Desgaste adhesivo con interacción plástica}

- La intensidad de desgaste Ih seráa ${ }^{[2]}$ :

$I_{\mathrm{h}}=\sqrt{2} \mathrm{C}\left(p_{\mathrm{a}} / \mathrm{HB}\right) \quad \Delta^{1+\beta_{\mathrm{r} / 1-\beta \mathrm{r}}}\left(k_{\mathrm{f}} / e_{\mathrm{o}}\right)^{1++/ 2} \eta_{\mathrm{c}, \mathrm{a}}^{\mathrm{t}-{ }^{1++\beta / 1-\beta}}$

siendo $K_{\mathrm{f}}=\sqrt{\left(\sigma_{\mathrm{el}}+2 f \cdot H B\right) /\left(\sigma_{\mathrm{el}}-2 f \cdot H B\right)}$

el número de ciclos, n, hasta el daño será:

$n_{\mathrm{c}}=\left[\left(2 r e_{\mathrm{o}} / d\right) \sqrt{\left.\left(\sigma_{\mathrm{el}}-2 f \cdot H B\right) /\left(\sigma_{\mathrm{el}}+2 f \cdot H B\right) v\right]^{t . K_{\mathrm{t}}}}\right.$

siendo d la media de los diámetros del contacto entre las superficies

$d=2\left(2 \alpha r R_{\text {máx }} \varepsilon / \nu\right)^{1 / 2}$

y

$$
K_{\mathrm{t}}=\frac{\Gamma(v+\mathrm{t} / 2)}{\Gamma(v) \Gamma(1+\mathrm{t} / 2)}
$$

- El área real de contacto Ar se podrá calcular según Tabor ${ }^{[3]}$ por la expresión

$A_{r}=C \frac{F_{n}}{P_{y 1}}$

calculándose C por la expresión (4).

Como se podrá apreciar, en los cálculos de $\psi$, no interviene en la fórmula la carga, siendo 
determinantes las propiedades superficiales y la topografía de las superficies. Está claro que si la topografía de la superficie es tal que inicialmente se produce flujo superficial, la interacción repetida de las superficies, alisa la topografía, principalmente en el período de asentamiento hasta que decrece la desviación standard de las alturas de las rugosidades s y/o el radio de curvatura de las asperezas se incrementa, y de esta forma el contacto pasa de plástico a elástico ${ }^{[1]}$

Si el desgaste no es adhesivo, el algoritmo discrimina si lo es abrasivo, y de ser así los cálculos se realizan según F4.

\subsection{F4: Desgaste abrasivo}

- El volumen de material desgastado, $V_{d}$, se calcula según Sarkar ${ }^{[4]}$ :

$$
V_{\mathrm{d}}=L(0,63 \operatorname{ctg} \alpha)(P / H)
$$

donde

$L$ es la trayectoria de fricción

$\alpha$ es la media del semiángulo de los vértices agu-

dos de las partículas abrasivas

$P$ es la carga aplicada

$H$ es la dureza del material identado

- La intensidad de desgaste $I_{\mathrm{h}}$ se puede calcular mediante las fórmulas

$I_{\mathrm{h}}=\Delta V / L$

ó

$I_{\mathrm{h}}=\Delta \mathrm{G} / \mathrm{L}$

y también

$I_{\mathrm{h}}=\Delta \mathrm{V} / \mathrm{t}$

ó

$I_{\mathrm{h}}=\Delta \mathrm{G} / \mathrm{t}$

donde

$\Delta V$ es la diferencia en volumen del elemento desgastado antes y después del proceso de desgaste para una trayectoria de fricción $L$ o un tiempo $t$ respectivamente.

$\Delta \mathrm{G}$ es la diferencia en peso del elemento desgastado antes y después del proceso de desgaste para una trayectoria de fricción $L$ o un tiempo $t$ respectivamente.

En (5), si el material es heterogéneo, puede calcularse $\mathrm{H}$ según la fórmula:

$1 / \mathrm{H}=\left(x_{1} \frac{1}{\mathrm{H}_{1}}+x_{2} \frac{1}{\mathrm{H}_{2}}+\cdots \cdots \cdot x_{\mathrm{n}} \frac{1}{\mathrm{H}_{\mathrm{n}}}\right)$

en la que

$x_{\mathrm{n}}$ es la fracción de cada fase presente en la estructura

$H_{n}$ es la dureza de cada fase

En estos casos, la coherencia fase-matriz juega un papel importante que puede alterar el resultado, por lo que debe tenerse en cuenta.

También se ha demostrado ${ }^{[5]}$ que si las partículas abrasivas son esféricas, la masa de metal desgastado, M, por unidad de trayectoria de fricción se halla según la fórmula:

$1 /(\mathrm{M} / \rho) \infty(H / N)^{3 / 2}$

donde

$\rho \quad$ Es la densidad del material que se desgasta

$H$ es la dureza del material que se desgasta

$N$ es la carga normal aplicada

Siguiendo el esquema, si el desgaste no es del tipo adhesivo ni abrasivo, se discrimina si es erosivo. En este caso los cálculos se realizan según el apartado siguiente.

\subsection{F5: Desgaste erosivo}

Un criterio que se emplea para categorizar el fenómeno del impacto en el desgaste erosivo es el número de daño $\mathrm{N}_{\mathrm{d}}$, que es el número de impactos necesarios para causar el desprendimiento de partículas del material impactado.

El número de daño $N_{\mathrm{d}}$ puede ser calculado según Johnson ${ }^{[6]}$ mediante la fórmula:

$N_{\mathrm{d}}=\rho v^{2} / \sigma_{\mathrm{y}} g$

donde

$\rho$ es la densidad del material impactado

$v$ es la velocidad relativa de impacto normal de las partículas erosivas

$\sigma_{y}$ es el esfuerzo de fluencia del material impactado $g$ es la aceleración de la gravedad. 
Mientras mayor sea la relación $\rho / \sigma_{y}$, para una velocidad de impacto dada, mayor será la resistencia del material al daño (a la erosión).

También puede calcularse el incremento de la temperatura, $\Delta T$, por la energía transferida en el impacto y que es absorbida por el material impactado. Esto es importante ya que $\mathrm{s}_{\mathrm{y}}$ depende de la temperatura. Así $\Delta \mathrm{T}$ es:

$\Delta T=v^{2} / 2 g c$

donde

c es el calor específico del material impactado.

El volumen del crater $V_{c}$, generado en el impacto, puede demostrarse que no depende de la forma de la partícula erosiva ${ }^{[4]}$ y puede ser calculado así:

$V_{c}=\left(1 / 2 m v_{i}^{2}\right) / \sigma_{y} g$

donde

$v_{\mathrm{i}}$ es la velocidad de impacto para una penetración cero de la partícula, o sea la inicial de impacto normal a la superficie impactada.

$m$ es la masa de la partícula erosiva.

La masa del material removido, $q$, en un proceso erosivo, es proporcional al cubo de la velocidad inicial de impacto $v_{\mathrm{i}}^{[4,7]}$, así q $\infty v_{\mathrm{i}}^{3}$. Por lo general, en la práctica, el exponente es inferior a 3 ya que ${ }^{[7]}$, al generarse el cráter por el impacto, no todo su volumen se desgasta.

El volumen erosionado, $V_{d}$, depende del ángulo con que impacta la partícula erosiva, $\alpha$ ). Este volumen puede ser calculado según Finnie ${ }^{[8]}$.

$V_{\mathrm{d}}=v_{\mathrm{i}}^{2} / 8 g \sigma_{\mathrm{y}}\left(\operatorname{sen} 2 \alpha-3 \operatorname{sen}^{2} \alpha\right)$

si $\alpha<18,5^{\circ}$

y

$V_{\mathrm{d}}=v_{\mathrm{i}}^{2} / 24 g \sigma_{\mathrm{y}}\left(\cos ^{2} \alpha\right)$

si $\alpha>18,5^{\circ}$

\subsubsection{Erosión por líquidos}

En la erosión causada por líquidos es importante conocer el valor de la presión en la interfase líquido-sólido.
Ya en 1932, Gardner demostró que era análogo el tratamiento a este fenómeno al que se produce en una colisión elástica entre dos sólidos, siendo uno de ellos una barra ${ }^{[9]}$.

Asumiendo una colisión normal, la presión p puede ser calculada por la fórmula:

$p=v(\rho D / g)^{1 / 2}$

donde:

$v$ es la velocidad de impacto

$D$ es el módulo de elasticidad cúbica del líquido, siendo $D=1 / k$ fórmula en la que

$k$ es el coeficiente de compresibilidad del líqui$\mathrm{do}^{[10]}$

El efecto de esta presión, que produce una onda acústica que se expande en el metal, genera rugosidades en lá superficie de éste, en los centros de las cuales, se produce la erosión al crecer la onda. El efecto no lo causa el impacto directo, si no un proceso de cavitación, al surgir burbujas de vapor, cuando la presión calculada, $p$, es menor que la presión de vapor del líquido, $p_{\mathrm{v}}$, a una temperatura dada:

$p<p_{\mathrm{v}}$

Si la presión sobrepasa el límite de fluencia del material, surgen las rugosidades.

Si el desgaste tampoco es, del tipo erosivo, se discrimina, entonces, si es un desgaste mecánico corrosivo . A su vez aquí se discrimina si el medio es oxígeno (o aire) u otro medio, obteniéndose en el primer caso la salida F6 y en el segundo la salida F7.

\subsection{F6: Desgaste mecánico corrosivo en pre- sencia de aire $u$ oxígeno}

Este tipo de desgaste, también denominado de oxidación, se lleva a cabo cuando el par de fricción está sometido a la acción de oxígeno del aire u oxígeno contenido en lubricantes.

En este tipo de desgaste influye, de forma determinante, el tipo de óxido que se forma, su espesor, tiempo de reposición y la relación entre su dureza y la del material base, siendo la velocidad de oxidación, $M$, función de la temperatura según la expresión:

$$
M \infty \exp (-\mathrm{a} / \mathrm{T})
$$


donde

a es una constante

$T$ es la temperatura

Esto, a su vez, considera dos posibilidades ${ }^{[11]}$ :

- 1)que la capa de óxido se forme y se destruya en forma continua.

- 2)que la capa de óxido se forme, alcance un espesor crítico y luego se destruya.

Para cada caso el desgaste $W$ se puede calcular según:

1) $W_{1} \sim \exp \left(-a^{\prime} / v\right)$, para velocidades de deslizamiento pequeñas

$W_{1} \sim \exp \left(-a^{\prime} / v^{1 / 2}\right)$, para altas velocidades de deslizamiento.

2) $W_{2} \sim \exp \left(-a^{\prime \prime} / v\right)$, para pequeñas velocidades de deslizamiento

$W_{2} \sim \exp \left(\mathrm{a}^{\prime \prime} / v^{1 / 2}\right)$, para altas velocidades de deslizamiento.

calculándose a' y a", en cada caso, a partir de (6), al obtenerse $\mathrm{M}$ por ensayos de laboratorio.

\subsubsection{F7: Desgaste mecánico corrosivo en diferentes medios}

El desgaste mecánico-corrosivo en otro medio que no sea oxígeno es quizás el mecanismo más complejo de desgaste y, hasta ahora, no se propone en la literatura una fórmula única para su cálculo.

En este tipo de desgaste influyen diferentes factores, entre otros

- tipo o tipos de óxidos que se forman y su relación con el material base

- posibilidad de formación de micropares galvánicos

- características y propiedades del material base

- velocidad de deslizamiento si el desgaste mecánico es adhesivo

- tipo y forma de partículas y la relación de sus propiedades con las de las capas de óxido si el desgaste mecánico es abrasivo

- tipo y forma de las partículas, ángulo de incidencia y velocidad de impacto si el desgaste mecánico es del tipo erosivo.
Este tipo de desgaste puede ser dividido en tres etapas $^{[12]}$.

- difusión de oxígeno hacia la superficie del cuerpo

- crecimiento de las capas de óxido

- destrucción de las capas.

Para determinar el desgaste en estos casos es indispensable realizar ensayos en condiciones reales. También se han obtenido resultados satisfactorios, modelando físico-matemáticamente el objeto real en condiciones de laboratorio ${ }^{[12]}$.

Por último, si el desgaste no se lleva a cabo en condiciones de fricción seca (ver algoritmo), siendo éstas lubricadas, se obtienen nuevas salidas, dependiendo de que se trate de lubricación seca, F8, lubricación total, F9, o lubricación mixta, F10. $\mathrm{Ya}$, si en este momento no se ha llegado a establecer el tipo de desgaste entre aquellos no lubricados, debe comenzarse de nuevo el análisis desde el principio, pues no existe otro tipo de clasificación. Por ello, en el esquema aparece la salida A que regresa al origen.

Al referirse a condiciones lubricadas, se piensa en cojinetes fundamentalmente. Estos, pueden ser parte integrante de un equipo, pero usualmente son un componente separado, que a su vez puede ser un buje, la mitad de un cojinete, una arandela tope $u$ otro tipo de superficie que, sirviendo de parte intercambiable, fusible y condicionada a desgastarse, interactúa con otro elemento en forma deslizante. Estos componentes, generalmente, se clasifican como de paredes delgadas, con espesor $\leq 5 \mathrm{~mm}$ y de paredes gruesas, si el espesor es $>5 \mathrm{~mm}$. Los cojinetes con diámetro $>150 \mathrm{~mm}$, se clasifican, por lo general, como de pared gruesa ${ }^{[13]}$.

$\mathrm{Al}$ analizar esta salida en el algoritmo es necesario tener en cuenta tres categorías de regímenes lubricantes:

- Lubricación límite o seca

- Lubricación completa (las superficies totalmente separadas por una capa lubricante)

- Lubricación mixta (la carga es soportada parcialmente por una capa lubricante y parcialmente las superficies entran en contacto).

\subsection{F8: Desgaste en cojinetes con lubricación seca}

Es posible calcular el calor $Q$, generado en el contacto para lubricación seca según Ron y Conway ${ }^{[14]}$ mediante la expresión 


$$
\mathrm{Q}=k \mathrm{~A}^{\prime}\left(T_{\mathrm{s}}-T_{\mathrm{a}}\right) / e=\mu \mathrm{PVA}
$$

donde

$T_{\mathrm{s}}$ es el incremento de temperatura en la superficie, por encima de la ambiente

$T_{\mathrm{a}}$ es la temperatura ambiente

$e$ es el espesor a través del cual fluye el calor (Fig.

2)

$A^{\prime}$ es el área por donde fluye el calor

$k$ es la conductividad térmica del material.

De la expresión (7) puede sacarse como conclusión que el calor generado, $Q$, es función de $P V$, donde $\mathrm{P}$ es la carga específica (presión) y $\mathrm{V}$ la velocidad de rozamiento.

A su vez,

$$
(P V)_{\text {máx }} \text { o } k / \mu \mathrm{e}\left(\mathrm{A}^{\prime} / \mathrm{A}\right)\left(T_{\mathrm{s}}-T_{\mathrm{a}}\right)_{\text {máx }}
$$

Hoy resulta común caracterizar la severidad del proceso de deslizamiento de cojinetes en función del factor PV para la etapa de trabajo de los mismos.

- El valor del desgaste $\mathrm{H} / \mathrm{t}$, para muchos materiales, es constante después del período inicial de asentamiento y puede expresarse como:

$$
H / t=K \cdot P \cdot V
$$

donde

$H$ es el espesor de desgaste

$t$ es el tiempo

$K$ es el valor de desgaste específico.

En (9) el valor del desgaste específico es el desgaste volumétrico dividido por la carga y la trayec-

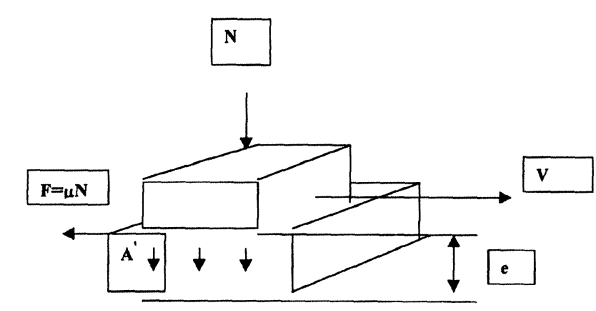

Figura 2. Factores en la interacción de dos elementos deslizantes en contacto.

Figure 2. Factors on the interactions of sliding elements in contact. toria de fricción. De esta forma, el desgaste específico permisible en un par de fricción $\mathrm{Hp}$ se representará con la fórmula:

$H_{p}=K \cdot P \cdot V \cdot t$

\subsubsection{Desgaste en cojinetes con lubricación}

La primera condición a tener en cuenta en estos cálculos es el valor de 1 , coeficiente que define si la lubricación es completa o mixta. El nomograma que se muestra en la figura 3 se emplea para calcular el valor de $h_{\min }$ que es el mínimo espesor de capa para lubricación total.

Para el uso adecuado del nomograma deben conocerse claramente los siguientes factores:

- diámetro interior del cojinete, $d$

- longitud de apoyo, $L$

- clarencia u holgura radial, $c_{\mathrm{r}}$

- longitud total del cojinete, $b$

- carga aplicada, $N$

- carga específica, $N / b \cdot d$

- grado de viscosidad según ISO, a $40^{\circ} \mathrm{C}$ ó $105^{\circ} \mathrm{F}$

- clarencia u holgura en diámetro $c_{\mathrm{d}}$

- relación de clarencia u holgura $c_{\mathrm{d}} / d$

Los valores típicos de $h_{\text {mín }} / c_{\mathrm{r}}$, para cojinetes hidrodinámicos de carga constante, están en el rango de 0,4 a $0,2^{[15]}$. Si esta relación aumenta es posible la fuga de lubricante para velocidades mayores de $300 \mathrm{rpm}$. Si la relación es inferior a 0,2 existe la posibilidad de contacto entre las superficies y el paso a un proceso de lubricación mixta.

El valor mínimo de capa lubricada $h_{\text {mín }}$ está en el rango de 0,0025 a 0,025. Este valor está relacionado con la suma de las alturas máximas de las asperezas en el eje, $e_{\mathrm{e}}$, y el cojinete, $e_{\mathrm{c}}$, dando lugar a la relación $\lambda$.

$$
\lambda=h_{\min } /\left(\sigma_{1}+\sigma_{2}\right)
$$

$$
\lambda=h_{\min } / \sqrt{\left(\sigma_{1}^{2}+\sigma_{2}^{2}\right)}
$$

ó

$$
\begin{aligned}
& \lambda=h_{\text {min }} /\left(R_{\mathrm{pm}_{1}}+R_{\mathrm{pm}_{2}}\right) \\
& \lambda=h_{\text {min }} / \sqrt{R_{\mathrm{pm}_{1}^{2}}^{2}+R_{\mathrm{pm}_{2}}^{2}}
\end{aligned}
$$

siendo:

$\mathrm{Rpm}_{\mathrm{n}}=1 / 5\left(R \mathrm{pm}_{\mathrm{n} 1}+R \mathrm{pm}_{\mathrm{n} 2}+\ldots . .+R \mathrm{pm}_{\mathrm{n} 5}\right)$ 


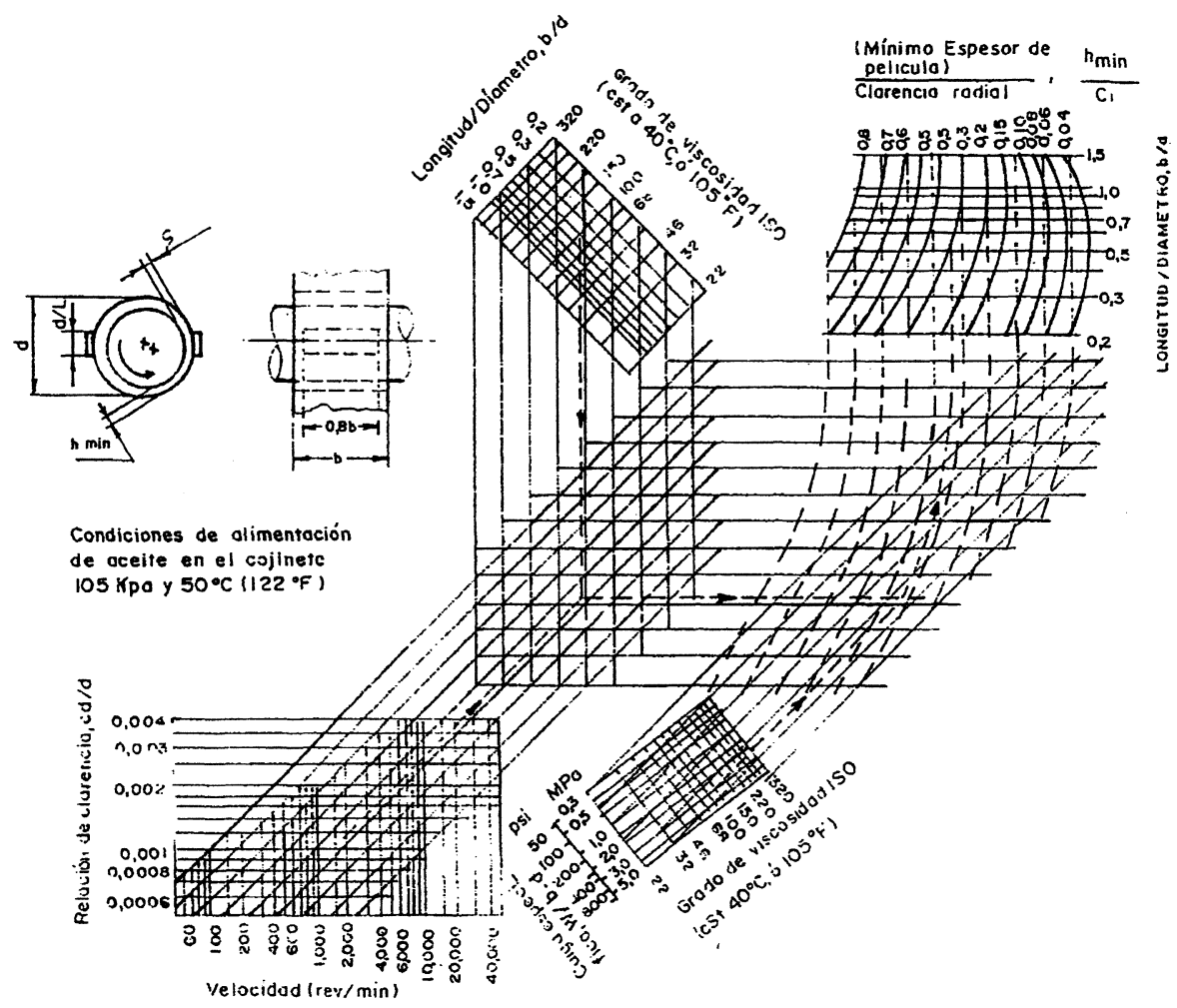

Figura 3. Nomograma para predecir el mínimo espesor de película de aceite en cojinetes con carga constante.

Figure 3. Nomogram for the prediction of the minimum oil film thickness on slide bearings under constant load.

siendo los subíndices 1 y 2 , en (10) y (11), los correspondientes a los dos cuerpos que forman el par tribológico.

Si el cálculo de $\lambda$ se hace según (10) el valor mínimo de $\lambda$ será 3 para diferenciar la lubricación total de la mixta y si se hace según (11) este valor será de 1 , o sea $\lambda>1$ para que la lubricación sea total.

\subsubsection{F9: Desgaste en cojinetes en condiciones de lubricación total o mixta}

En estos tipos de lubricación no es posible calcular un valor de desgaste, ya que éstos tienen lugar solo en el proceso de arranque y parada del par de fricción o producto del deterioro o pérdida de lubricante, contaminación de éste o presencia de partículas abrasivas, bien del propio elemento desgastado o del medio ambiente. Por lo tanto, el cálculo aquí consiste en garantizar la capa de lubricante mínima necesaria $\left(h_{\mathrm{mín}}\right)$ para prevenir el deterioro de la superficie y después comprobar si se cumple el valor de $\lambda$ para esta condición.

En este tipo de lubricación, según se explicó más arriba, la carga es soportada parcialmente por la capa lubricada y, parcialmente, por las superficies que entran en contacto. Esto origina un incremento en la fricción y en la temperatura, que puede llegar a ser 10 ó 50 veces mayor ${ }^{[16]}$.

El tiempo de trabajo en este tipo de lubricación debe ser nínimo. El valor de $\lambda$ desciende por debajo de los valores permisibles y las asperezas incrementan el valor de la carga que parcialmente soportaban. La pérdida de potencia $H$, que tiene lugar a partir de los incrementos de la carga o la reducción de velocidad, puede ser calculada por el nomograma que se muestra en la figura 3 . Este nomograma también puede emplearse para condiciones de lubricación completa o total.

\section{CONCLUSIONES}

- En el trabajo se ofrece un algoritmo que permite efectuar cálculos de desgaste a partir de discriminar en el tipo de desgaste que tiene lugar.

- El algoritmo sintetiza las principales formulaciones para el cálculo del desgaste existente en la literatura especializada. 
Lista de símbolos

$\mathrm{A}_{\mathrm{a}} \quad$ Area aparente de fricción.

$A_{r} \quad$ Area real de fricción.

b Parámetro que caracteriza la curva de la superficie de apoyo.

$\beta \quad$ Media de los radios de las asperezas de una superficie.

$\theta \quad$ Semiángulo del vértice en partículas abrasivas.

$\delta \quad$ Alargamiento expresado en porcentaje.

E Módulo de elasticidad del material.

f Coeficiente de fricción.

$\Gamma \quad$ Función gamma.

g Aceleración de la gravedad.

$\mathrm{h}_{\text {mín }}$ Espesor mínimo de capa lubricante para garantizar lubricación total en cojinetes.

H Dureza.

$i_{h} \quad$ Intensidad específica de desgaste.

Ih Intensidad de desgaste.

$\mathrm{k}$ Conductividad térmica.

L Trayectoria de fricción.

$\lambda$ Factor que permite determinar el espesor de capa mínima permisible para un trabajo estable bajo un régimen de carga dado en superficies lubricadas y fundamentalmente en cojinetes.

$\mu \quad$ Coeficiente de Poisson.

n Número de ciclos hasta el daño en un proceso de fatiga.

$\mathrm{N}$ Carga normal.

$v \quad$ Parámetro que caracteriza la aproximación de la curva que describen las rugosidades sobre una superficie de apoyo.

$\psi \quad$ Índice de plasticidad.

Q Calor generado por fricción.

$\mathrm{Ra}$ Media aritmética de la desviación del perfil de una superficie.
$\mathrm{R}_{\text {máx }}$ Altura máxima de las rugosidades de una superficie.

Rp Altura media de las rugosidades de una superficie.

$\sigma \quad$ Rugosidad rms (raíz cuadrada de la media del cuadrado de las desviaciones de la altura de las rugosidades de una superficie).

$\sigma_{\mathrm{el}} \quad$ Límite elástico de un material.

$\sigma_{\mathrm{y}} \quad$ Límite de fluencia de un material.

\section{REFERENCIAS}

[1] J. LARSEN-BASSE Basic Theory of solid friction., NSF, Friction, Lubrication and Wear Technology, Vol.18, ASM, 1992.

[2] I.V. KRAgelski y V.S. Kombalov, Mashinoestroenie (1977).

[3] D.A. TABOR, Wear 32 (1975).

[4] A.D. SARKAR, Friction and wear, Academic Press Inc., London, 1980.

[5] P.J. Alison, M.F. Stroud y H. Wilman, Proc. Inst. Mech. Eng. London 179 (1964) 246.

[6] W. JOHNSON, Impact strength of materials, Edward Arnold, London, 1972

[7] G.L. SHeldon y A. KANHERE, Wear 21 (1972) 195

[8] I. FinNiE, Wear 3 (1960) 87-103.

[9] F.W. GARDNER, The engineer, 1932

[10] E.A. Shtrauf, Fundamentos de la Física Molecular, Camajo, Barcelona, España, 1976.

[11] T.F. Quinn, Br. J. Appl. Phys. 13 (1962).

[12] F.F. TAO, ASLE Trans. 12 (1969).

[13] R. Reinoza, Tesis Doctoral, La Habana, Cuba, 1999.

[14] P. Ron y J.M. CONWAY Jones, Glacur Vander Vell Inc., vol 18, ASM, 1992.

[15] K.C. LUDEMA Failures of sliding bearings. Failure analysis and prevention, vol. 18, Metals Handbook, ASM, 1986.

[16] H. Christensen, Wear 17 (1971) 149-162. 